\title{
Zukunftsfach Radiologie: Dreh- und Angelpunkt von Diagnose und Therapie
}

Krise als Chance: Covid-19 hat erstmals zu einheitlichen Standards für Lungen-CTs an allen deutschen Universitätsklinika geführt. Mit dem Projekt RACOON (Radiological Cooperative Network), gefördert durch das „Netzwerk Universitätsmedizin (NUM)“ wurden so Grundlagen für gemeinsame Datenpools, Vernetzung und KI geschaffen. Wie muss jetzt die Zukunft der Radiologie weiter gestaltet werden, welche einmaligen Chancen bieten sich? Wie sieht die Rolle der Radiolog*innen von morgen aus? Welche Eigenschaften sollten Medizinstudent*innen und junge Kolleg*innen mitbringen? Und warum ist die Rolle der ModeratorIN und KommunikatorIN in der Radiologie so wichtig? Darüber sprechen wir in der zweiten Folge des Jubiläumspodcasts „100 Jahre RöFo \& DRG“ mit Professor Konstantin Nikolaou, Ärztlicher Direktor der Abteilung für Diagnostische und Interventionelle Radiologie am Universitätsklinikum Tübingen, President-elect der Deutschen Röntgengesellschaft (DRG) und Schriftleiter der RöFo. Die Fachzeitschrift, die seit 125 Jahren bei Thieme erscheint, ist seit 1922 das offizielle Organ der DRG. Lesen Sie hier einen Ausschnitt des Gesprächs.

\section{Welche besonderen Fortschritte hat die Radiologie während der Coronapande- mie gemacht?}

Hier möchte ich exemplarisch das Projekt RACOON (Radiological Cooperative Network) nennen, das 2020 von Professor Hamm, Berlin, und Professor Vogl, Frankfurt, sowie von weiteren PartnerInnen ins Leben gerufen wurde. Alle radiologischen Abteilungen der 36 Universitätsklinika des Landes sind daran beteiligt. Die Projektpartner haben sich darauf geeinigt, Bilder von mit SARS-CoV2-infizierten Lungen und von Verdachtsfällen nach gemeinsamen Protokollen und Standards zu erstellen. Dadurch kann eine große Datenmenge in einheitlicher Befund- und BildQualität generiert werden. Auf dieser Basis lassen sich dann auch neue Technologien wie KI-Algorithmen zur KrankheitsDetektion und Verlaufs-Prädikation etablieren. Um für diese und zukünftige Pandemien besser gerüstet zu sein, sollen damit in Zukunft große Datenmengen auch mithilfe von $\mathrm{KI}$ - schneller analysiert und nach Auffälligkeiten durchsucht werden können. Das ist in dieser Form sicherlich ein Novum, das sich so viele wissenschaftliche radiologische Partner gemeinsam auf ein Procedere und eine gemeinsame Datensammlung festlegen.

Ich glaube, diese breite Kooperation auf dieser innovativen technischen Plattform ist etwas, wovon die radiologische Forschung längerfristig profitieren wird, auch für andere wissenschaftliche Fragestellungen. Dieses Netzwerk ist ein Standard, um auch in Zukunft kooperative Forschung, Netzwerk-Forschung, zu betreiben - mit bestimmten Qualitäts- und technischen Kriterien. Auslöser war tatsächlich leider die Pandemie. Oder dann zum Glück in dem Fall zumindest für das, was bleibt.

\section{Was haben Sie konkret über COVID-19 und das Zielorgan Lunge gelernt?}

Wir haben zunächst Lungen-Datensätze von CTs mit einem bestimmten festgelegten Protokoll aufgenommen. Wir haben uns geeinigt, wie man diese befundet, welche Parameter und Merkmale im Bild man berichtet, in welchen Kategorien man diese einteilt etc. Dies gelang in enger Kooperation mit verschiedenen Fachgesellschaften, wie auch mit der

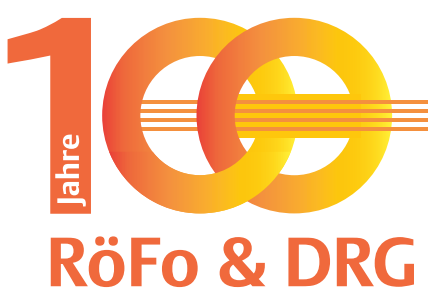

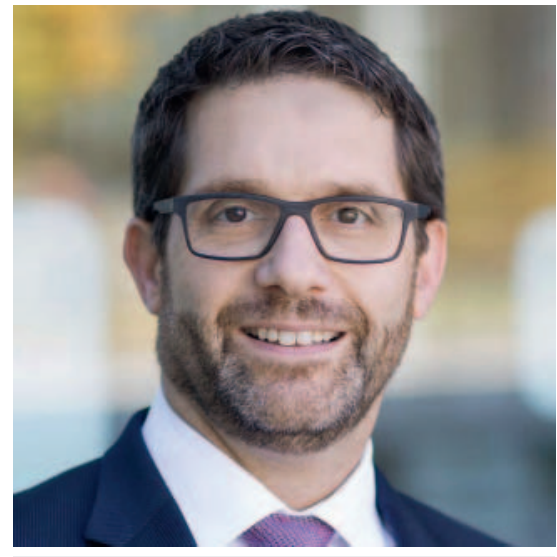

Prof. Konstantin Nikolaou

Arbeitsgemeinschaft Thoraxradiologie der DRG. Durch diesen Aufwand ergibt sich eine strukturierte Datenbasis. Diese umfasst sowohl die Befunde, als auch die Annotation, also eine Nachbearbeitung und Markierung der Bilder und Befunde. Dies bildet die Grundlage, um darauf Algorithmen der KI zu trainieren. Zum Beispiel einen Algorithmus, der primär die Infiltrate erkennt, der sie vielleicht differenziert von anderen, nicht COVID-typischen Infiltraten oder ein Algorithmus, der anhand der Bildbefunde und Parameter versucht vorherzusagen, wie schwer der Krankheitsverlauf sein wird.

Denn das ist natürlich eine der zentralen Fragen: Welche Veränderungen im Lungen-CT sind prognostisch wertig und wirksam? Was bedeutet das für Patientlnnen? Wie erhöhen sich Risiken für einen schweren Verlauf, für eine Hospitalisierung oder auch für eine Intensiv-Behandlung?

Ist es in dem Zusammenhang auch wichtig, dass Sie sich von vornherein in die Studienplanung anderer medizinischer Fachdisziplinen einbringen - etwa, damit auch dort die für Sie wichtigen bildgebenden Parameter nach Ihren Standards miterfasst werden?

Ich denke, das ist ein ganz wichtiger Hinweis, wie wir die Daten auch zwischen den Fächern verknüpfen. Die Daten, die wir generieren, sind natürlich auch auszu- 
tauschen mit den klinischen und anamnestischen Patient*innen-Daten und damit unbedingt auch zu korrelieren. Es gibt im "Netzwerk Universitätsmedizin (NUM)“ andere geförderte Großprojekte, die eben genau das machen. Sie bauen eine klinische Datenbasis auf, sammeln Daten von Patient*innen, nehmen auch hier Verläufe auf und korrelieren sie mit eben diesen. Das ist sicherlich ein wichtiges Ziel dieser Netzwerkstrukturen und Projekte im NUM, dass man sich abspricht, um prospektiv auch Daten gemeinsam zu erheben.

Wie stehen Sie insgesamt zur Entwicklung von Algorithmen und $\mathrm{KI}$ in der Radiologie?

Ich glaube, wir sollten hier als Vertreter*innen unseres Faches, der Radiologie, so wie wir es jetzt mit RACOON auch wieder sind, technischer Vorreiter sein. Wenn man mit digitalen, radiologischen Daten, aber auch mit komplementären klinischen Metadaten arbeitet, sollte man wissen, wie man diese technisch richtig zusammenbringt, wie man sie richtig auswertet, wie man Algorithmen richtig darauf trainiert und Standards entwickelt, die dann auch für eine Nutzung in der klinischen Routine validier- und zertifizierbar sind.

Die Implementierung der KI wird unser tägliches Arbeiten unterstützen. Wir sollten diese neue methodische Qualität aufnehmen und nutzbar machen und durch die Beantwortung auch neuer, komplexerer Fragen einen klinischen Mehrwert erzeugen.

Und solange die Radiologie selbst die Infrastruktur und die Methodik (mit-)entwickelt, sind wir genau da, wo wir sein wollen, nämlich in dem Fall als wissenschaftliches Fach, als Vorreiter einer Entwicklung, die neue Methoden klinisch nutzbar macht.

\section{Brauchen wir zukünftig weniger oder nur andere Radiolog*innen?}

Ich glaube nicht, dass wir in Zukunft weniger Radiolog*innen brauchen. Ich glaube aber, dass es in unserem Alltag genug Arbeitsschritte gibt, von denen wir uns wünschen würden, dass sie unterstützt oder zum Teil „automatisiert“ würden. Ich denke dabei an eine Vielzahl von Thorax-Röntgenaufnahmen, zum Beispiel Kontroll-Aufnahmen in einem längeren klinischen Verlauf. Hier nehmen Sie einen unterstützenden Algorithmus, der beispielsweise bestimmte auffällige Befunde in Ihrer Arbeitsliste priorisiert, durchaus dankend an. Ich glaube aber, dass die Arbeit in dem Umfeld komplexer DatenModellierung sehr viel neue Kapazitäten und auch neues Wissen erfordern wird. Nehmen Sie das Beispiel der prädiktiven KI-Algorithmen. Sie erweitern gerade unser diagnostisches Feld und erfordern eine enge Korrelation mit genetischen, pathologischen und weiteren diagnostischen Daten. Wir werden uns in die Richtung einer diagnostisch breit aufgestellten, therapeutisch beratenden und interventionell tätigen Radiologie entwickeln, mit ärztlichen Mitarbeiter*innen, die dieses komplexe Umfeld verstehen. Wir werden also zukünftig breiter und komplexer denken müssen, vor allem in der diagnostischen Interaktion mit den Nachbarfächern wie der Pathologie und zunehmend der Genetik. Es wird ebenso darum gehen, im Kontext mit diesen Fächern auch schwierigere klinische Entscheidungen zu treffen, etwa in der Indikation neuer molekularer Therapien oder Immuntherapien in der Onkologie.

In diesem Zusammenhang sind beispielsweise molekulare Tumor-Boards zu nennen. Hier liegen uns zunehmend neuartige molekulare und genetischen Informationen und diagnostische Methoden vor, die jetzt auch in die Regelversorgung übergehen. Und wir müssen lernen, wie zum Beispiel bestimmte Bildparameter, Imaging Biomarker, mit genetischen Informationen korrelieren und sich ergänzen. Da müssen wir alle in unserem Fach viel dazulernen. Es wird, glaube ich, eine sehr spannende und interessante Zeit!

Zusammenfassend bin ich der Meinung, dass wir in unserer täglichen Arbeit den Fokus wechseln, zunehmend in die interdisziplinäre Kommunikation und Diskussion sowie in die Integration komplexer Informationen gehen werden, neben der Weiterentwicklung und Verbreitung interventioneller radiologischer Methoden.
Welche Eigenschaften sollten Medizinstudierende oder junge Kolleg*innen mitbringen, wenn sie sich für die Radiologe interessieren?

Auf der einen Seite sind wir traditionell ein technisch getriebenes Fach. Man sollte also Interesse haben an der technischen Machbarkeit und an den Entwicklungsmöglichkeiten neuer Bildgebungsmethoden. Dazu gehören Offenheit für die Digitalisierung und auch die Anwendung von mathematischen und KI-Algorithmen. Selbst programmieren können, muss man nicht zwingend, aber ein technisches und mathematisches Grundverständnis sind sicherlich von Vorteil.

Neben dem technischen Interesse empfinde ich es als für unseren Beruf ganz zentral, dass man die täglich gelebte und praktizierte klinische Vielfalt liebt. Sicherlich kann man sich am Ende seiner Ausbildung durch Subspezialisierung in einem bestimmten Bereich vertiefen und auch in diese Richtung kontinuierlich weiterentwickeln, aber Sie müssen die medizinische Breite lieben. Sie sehen und erleben täglich eine unglaubliche und spannende Vielzahl von Pathologien und Fragestellungen. Und ich glaube, und das unterschätzt man vielleicht, Sie sollten als Radiolog*in ein guter Kommunikator sein. Denn Sie sind in einem klinischen Umfeld, in welchem man ständig spricht, diskutiert, berät. Und diese Rolle der Moderator*in und Kommunikator*in muss einem liegen.

Radiologie ist nicht nur theoretisch, sondern auch praktisch. Radiolog*innen führen interventionelle Eingriffe durch.

Absolut. Und ich glaube, wenn wir über das Zukunftsbild der Radiologie sprechen, ist das auch etwas, was man als Kolleg*in mitbringen muss. Denn die interventionelle Radiologie und die minimal-invasive bildgesteuerte Therapie werden ihre Rolle weiter ausbauen und zentraler Bestandteil von sehr komplexen, multidisziplinären Therapien sein. In enger Zusammenarbeit mit anderen Fachdisziplinen wird es darum gehen, an der richtigen Stelle die richtige Methode - medizinisch, chirurgisch oder eben die radiologisch interventionelle anzuwenden. Das sieht man auch an der Erweiterung von Indikations-Spektren, an 
neuen Interventionsmöglichkeiten und neuen minimal-invasiven Techniken. Auch hier spielt die Digitalisierung eine wichtige Rolle. Wir arbeiten an Methoden, wie man zum Beispiel auch intraprozedural Pathologien und Interventionen besser steuert und visualisiert, wie man in Zukunft z. B. mit 3D-Brillen und VR-Techniken in Operationen oder Interventionen durch Überlagerung diagnostischer Bilder besser den Interventions-Situs darstellen und darin navigieren kann.

Andere Fachgebiete, etwa die Kardiologie, sind auch interventionell tätig. Wie grenzt sich die Radiologie denn da ab?

Ich glaube, die Radiologie hat klar beschriebene Einsatzbereiche ihrer interventionellen Tätigkeit und diese sind auch gut etabliert und unstrittig. Es gibt bestimmte Schnittstellen oder Eingriffe und Interventionen, die traditionell von verschiedenen Fächern durchgeführt werden (könnten). Die Qualität, die dabei geliefert wird, sollte am Ende entscheiden. Für bildgestützte Interventionen gibt es klare Qualitätskriterien, Register und Berichtssysteme, die wir umfänglich bedienen und die den hohen Stellenwert unseres Faches in allen interventionellen Bereichen belegen.

Entscheidend für den Bereich der radiologischen Interventionen ist auch, dass wir technische Vorreiter sind, die richtigen Studien durchführen und unsere Mitarbeiter*innen exzellent ausbilden. Solange wir die Qualität und Versorgungsbreite hochhalten, wird die Radiologie hier immer ihren zentralen Stellenwert und ihre Rolle in den bildgesteuerten Interventionen behalten.

Innerhalb der DRG sind Sie Wissenschaftskoordinator. Warum hat die DRG diese Position geschaffen?

Zum einen muss die Radiologie als wissenschaftliches Fach wahrgenommen werden, weil sie ein solches ist. Nur ein wissenschaftlich fokussiertes Fach wird dauerhaft überleben, weil es selbst Treiber von Informationen und Technologien ist.
Mit der Wissenschaftskoordination als kooptierte Funktion im Vorstand der DRG sollen frühzeitig Entwicklungen aufgegriffen und vorangetrieben werden, sowie die Wissenschaftskommunikation und wissenschaftliche Kollaboration gefördert werden.

Themen, die von Seiten der Wissenschaftskoordination bearbeitet werden, sind etwa die Kommunikation und Kooperation mit wissenschaftlichen Institutionen wie der DFG oder dem BMBF, oder die Hilfe bei regulatorischen wissenschaftlichen Fragestellungen. Beispielhaft genannt sei hier die Wissenschaftsorganisation und Handhabung von wissenschaftlichen Projekten im Rahmen des neuen Medizinprodukterecht-Durchführungsgesetzes (MPDG). Es geht aber auch um konkrete inhaltliche Fragen. Bei der KI etwa müssen wir uns überlegen, nach welchen Standards und Kriterien das richtig gemacht wird. Ein weiteres wichtiges Feld ist wie schon dargestellt die wissenschaftliche Netzwerkbildung in dieser Pandemie, die Vernetzung von Themen und Daten und die Nutzbarmachung komplexer digitaler Inhalte.

Das sind Themen, die wir in unserem Fach genuin mit am besten beherrschen. Es sollte daher auch immer so sein, dass die Radiologie ganz bewusst eine wissenschaftliche Vorreiterrolle für bestimmte Themen annehmen sollte.

Dafür sind so ein Forum und eine Funktion gut geeignet, um sich auch mal die Zeit zu nehmen, strategisch zu denken, zu sagen „Was geht in die Translation?“, „Was kommt als nächstes an die klinische Front?“, „Was müssen wir selbst in die Hand nehmen?“, „Welche Rolle wollen wir in der Entwicklung spielen?". Solche Überlegungen müssen bewusst angestellt und vorangetrieben werden. Deswegen bin ich überzeugt, dass diese Funktion Wissenschaftskoordinator*in Sinn macht und auch in Zukunft weiterentwickelt werden sollte.
Sie sind nicht nur Wissenschaftskoordinator, sondern auch Schriftleiter der RöFo - und haben auch ansonsten viele Bälle in der Luft, viele Aufgaben und Ämter. Sie erhalten sicherlich laufend Angebote für weitere Aufgaben. Wie entscheiden Sie, wo Sie sich engagieren?

Ich weiß nicht, ob ich der Beste bin, um Tipps in dieser Hinsicht zu geben. Mein privates Umfeld würde sagen, das könnte man noch besser stratifizieren. Ich glaube, man muss sich der Frage stellen, warum man eine bestimmte Aufgabe oder Funktion machen bzw. übernehmen will.

Ist wirklich eine genuine Motivation da, ein Thema zu entwickeln? Hat man die Valenz und kann man die Aufgabe in einer ausreichenden Qualität erfüllen? Ich könnte mir angewöhnen, einmal mehr nein zu sagen, wenn ich ein wenig selbstkritisch sein darf. Ich gebe mir aber natürlich Mühe, die übernommenen Funktionen so gut wie möglich zu füllen.

Auf der anderen Seite gibt es auch Aufgaben und Inhalte, die einfach wichtig sind, sei es für unser Fachgebiet, unsere Fachgesellschaft, die eigene Universität oder die eigene Abteilung. Hier muss man auch Verantwortung übernehmen. Ich denke, das gehört zu unserem Beruf und Aufgabenspektrum.

Erfahren Sie mehr über weitere Themen im zweiten Teil des Jubiläumspodcasts „100 Jahre RöFo \& DRG“. Im Jubiläumsjahr sprechen wir in unregelmäßigen Abständen mit engagierten AkteurInnen und Mitgliedern der DRG über aktuelle Themen in der Radiologie. Sie finden diesen und weitere Beiträge auf www. thieme.de/roefo-pod. Oder scannen Sie einfach den QR-Code ein.

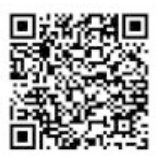

Das Gespräch führte Dr. Adelheid Liebendörfer, Thieme Communications. 